\title{
First-principles calculations of structural and electronic properties of monoclinic hafnia surfaces
}

\author{
Atashi B. Mukhopadhyay, Javier F. Sanz,* and Charles B. Musgrave \\ Department of Chemical Engineering, Stanford University, 380 Roth Way, Stanford, California 94305, USA
}

(Received 10 November 2005; revised manuscript received 31 January 2006; published 24 March 2006)

\begin{abstract}
We have carried out a systematic theoretical study of the surfaces of monoclinic hafnia $\left(\mathrm{HfO}_{2}\right)$ using plane waves and density functional theory based on the generalized gradient approximation. The fully relaxed structures of the bulk phases of $\mathrm{HfO}_{2}$ are found to be in excellent agreement with experimental data, the monoclinic phase being the most stable. Simulations of the monoclinic phase surfaces indicate a large relaxation which reduces the total surface energy of all nine faces considered by between $23 \%$ and $36 \%$, with a strong correlation between the unrelaxed and relaxed surface energies. Our calculations predict that the $(\overline{1} 11)$ and (111) faces of the monoclinic phase have the lowest surface energies and are hence the most stable faces. An analysis of the total and partial electronic density of states of bulk monoclinic $\mathrm{HfO}_{2}$ reveals that the outer valence band significantly mixes the $\mathrm{O} 2 p$ and $\mathrm{Hf} 5 d$ atomic states indicating some covalency of the Hf-O bonds. The total density and partial density of states of the monoclinic surfaces exhibit a surface state corresponding to the surface $\mathrm{O} 2 s$ states in the inner valence band region.
\end{abstract}

DOI: 10.1103/PhysRevB.73.115330 PACS number(s): 68.35.Md, 68.47.Gh, 73.20.At, 81.15.Gh

\section{INTRODUCTION}

An intense search for a new high dielectric constant material to replace $\mathrm{SiO}_{2}$ gate dielectrics in metal-oxidesemiconductor field-effect transistors, such as $\mathrm{HfO}_{2}$, has been undertaken to obtain insulating films with higher capacitance than thin $\mathrm{SiO}_{2}$ films and with greater thicknesses to resist tunneling leakage. ${ }^{1-4}$ Hafnium dioxide $\left(\mathrm{HfO}_{2}\right)$ has emerged as an excellent candidate due to its relatively high dielectric constant, wide band gap and stability on Si. The thermal stability of high- $k$ materials in direct contact with $\mathrm{Si}$ and $\mathrm{SiO}_{2}$ has become a key criterion in selecting suitable high $k$ choices. In addition to its potential use as a gate dielectric, $\mathrm{HfO}_{2}$ is also used as an optical coating because of its high refractive index and high transmission coefficient. ${ }^{5,6}$ Additionally, $\mathrm{HfO}_{2}$ exhibits a relatively high laser damage threshold due to its high melting point, thermal and chemical stability and wide transparent range from the infrared to ultraviolet. ${ }^{7,8}$ Furthermore, $\mathrm{HfO}_{2}$ serves as an excellent protective coating due to its thermal stability and hardness. ${ }^{9,10}$

Determination of stable surface structures of materials is required for the $a b$ initio prediction of material properties as the relative stability of the surfaces will affect the orientations and sizes of crystallites, which in turn affect materials properties. This is especially true for the prediction of surface properties such as surface reactivity. $\mathrm{HfO}_{2}$ thin films can be deposited on a variety of substrates using various methods. Techniques being explored include atomic layer deposition (ALD),$^{11}$ ion beam assisted deposition ${ }^{12}$ and jet vapor deposition. ${ }^{13}$ The structure of as-deposited $\mathrm{HfO}_{2}$ films is not yet fully resolved. For instance, various experimental studies found that $\mathrm{HfO}_{2}$ films deposited by ALD at $300{ }^{\circ} \mathrm{C}$ using $\mathrm{HfCl}_{4}$ and $\mathrm{H}_{2} \mathrm{O}$ as precursors are polycrystalline, and mainly consist of the monoclinic phase with the (111) surface exhibited as the main growth face. ${ }^{14,15}$ Upon annealing the intensities of the monoclinic peaks increase in the x-ray diffraction (XRD) spectra with the (111) and (111) monoclinic surfaces primarily populated. ${ }^{14}$ On the other hand, Aarik et al. reported that reflections from the (002), (200), and (111) monoclinic surfaces at $300{ }^{\circ} \mathrm{C}$ displayed the highest intensities rather than the (111) surface and that $(\overline{1} 11)$ reflections were weak or absent in the plane in which the XRD pattern was recorded, including at elevated temperatures. ${ }^{16,17}$ In yet another ALD deposited film, Kukli et al. report that the film grown at low temperature shows only traces of crystallinity in the XRD pattern. ${ }^{18}$ However, reflection high-energy electron diffraction of the same film revealed (002) and (104) orientations of monoclinic phases. However, for films grown at higher temperature $\left(750{ }^{\circ} \mathrm{C}\right)$, the (011), (111), and (111) diffraction peaks are strongest. Hence, direct comparisons to measured surface properties are difficult due to varying experimental conditions and film preparation methods. These experimental data thus only provide indirect and incomplete information about the detailed surface atomic structure. Moreover, these difficulties in interpretating different experimental studies are complicated by finite-size effects because samples are often polycrystalline, polymorphic, and contain impurities and dopants.

$\mathrm{Zr}$ and $\mathrm{Hf}$ are remarkably similar elements and consequently the surface properties of $\mathrm{HfO}_{2}$ are expected to be comparable to those of $\mathrm{ZrO}_{2}$. The surface properties of $\mathrm{ZrO}_{2}$ have been thoroughly investigated theoretically. ${ }^{19}$ Comparisons between our predicted properties for $\mathrm{HfO}_{2}$ do show many similarities to those predicted for $\mathrm{ZrO}_{2}$, although we also find significant quantitative differences in the calculated properties.

To the best of our knowledge, this contribution is the first theoretical investigation of the structural and electronic properties of $\mathrm{HfO}_{2}$ surfaces. In this paper, we have investigated the surface properties of low-index surfaces of the most stable (under the condition of atmospheric pressure and room temperature) form of $\mathrm{HfO}_{2}$, that is, the monoclinic surfaces. We present surface energies and near surface ionic relaxations. We have also performed a detailed examination of the electronic structures of the nine inequivalent monoclinic sur- 
faces to offer a more complete understanding of the stable $\mathrm{HfO}_{2}$ phases.

This paper is organized as follows. The next section describes the computational aspects of this study. In Sec. III we discuss results pertaining to the bulk properties, the surface structures and ionic relaxation and surface electronic properties. In the last section we draw some general conclusions.

\section{COMPUTATIONAL DETAILS}

In order to model the extended nature of the surfaces, density functional theory (DFT) calculations under periodic conditions were carried out using the Vienna ab initio simulation package (VASP). ${ }^{20,21}$ In these calculations the energy was calculated using the PW91 generalized gradient approximation (GGA) implementation of DFT proposed by Perdew et al. ${ }^{22,23}$ with the electronic states expanded using plane waves as basis set. The calculations were performed utilizing the projected augmented wave approach ${ }^{24}$ implemented in the VASP code. For Hf atoms, the semicore $5 p$ electrons have also been included in addition to the $5 d^{2}$ and $6 s^{2}$ valence electrons because we found that explicitly including the $5 p$ electrons is required in order to obtain correct lattice parameters for bulk $\mathrm{HfO}_{2}$. For $\mathrm{O}$ atoms $2 s$ and $2 p$ electrons were included. The plane-wave cutoff energy is $450 \mathrm{eV}$. The $k$ points were generated using the Monkhorst-Pack method and $4 \times 4 \times 4$ and $4 \times 4 \times 1$ grid sizes were used for structural optimization of the bulk and surfaces, respectively. For calculation of electronic properties the grid sizes were increased to $10 \times 10 \times 10$ and $10 \times 10 \times 1$, for the bulk and surfaces, respectively.

Forces on the ions were calculated using the HellmannFeynman theorem as the partial derivatives of the free electronic energy with respect to the atomic positions, and adjusted using the Harris-Foulkes ${ }^{25}$ correction to the forces. This approach for calculating the forces allows a geometry optimization using the conjugate-gradient scheme. Iterative relaxation of atomic positions was stopped when the change in total energy between successive steps was less than $0.001 \mathrm{eV}$. With this criterion, forces on the atoms were generally less than $0.1 \mathrm{eV} / \AA$.

To model the monoclinic surfaces we employed the wellknown slab approach consisting of a supercell that includes a portion of vacuum. After being replicated in the three directions of space, an array of slabs separated by a vacuum is obtained. The vacuum width was wide enough to prevent layer-to-layer interactions and we found that a width of $6 \AA$ was enough to ensure that the energy was converged to within $0.001 \mathrm{eV} /$ atom. The influence of slab thickness on the suitability of the surface models is crucial and will be analyzed in the discussion section.

\section{RESULTS AND DISCUSSION}

\section{A. Bulk properties}

To check the reliability of the computational approach we apply it to calculate measured, well-known properties of the bulk phases of $\mathrm{HfO}_{2}$. At room temperature the equilibrium phase of $\mathrm{HfO}_{2}$ is monoclinic which has the lowest free en- ergy of formation and the largest volume. ${ }^{26-28}$ At approximately $1300 \mathrm{~K}$ monoclinic $\mathrm{HfO}_{2}$ transforms into the tetragonal structure and transforms to the $\mathrm{CaF}_{2}$ cubic structure near $2700 \mathrm{~K} .{ }^{28}$ Here we fully relax both the cell lattice parameters as well as the ion positions. Table I reports our calculated structural parameters for the three phases of $\mathrm{HfO}_{2}$ we consider along with the energy of the system per $\mathrm{HfO}_{2}$ formula unit. We also include the data calculated in previous DFT studies by Zhao and Vanderbilt ${ }^{29}$ using ultrasoft pseudopotentials. We find that our values are in excellent agreement with the experimental results. In some cases and, especially for densities, our GGA results match experiment better than that of previous GGA results. We also calculate that the heat of formation of the monoclinic crystal, which is the most stable phase, is $-1070.5 \mathrm{~kJ} / \mathrm{mol}$, in good agreement with the experimental value of $-1144.7 \mathrm{~kJ} / \mathrm{mol}(7 \%$ error $){ }^{30}$ Our energy per formula unit reproduces the correct energetic ordering of the phases $\left[\mathrm{E}_{\text {MONOCLINIC }}\left(\mathrm{HfO}_{2}\right)\right.$ $<$ E TETRAGONAL $\left._{\text {TfO }}\left(\mathrm{HfO}_{2}\right)<\mathrm{E}_{\text {CUBIC }}\left(\mathrm{HfO}_{2}\right)\right]$.

\section{B. Surface structure}

The ability of our approach to reproduce the experimental results for the bulk properties of $\mathrm{HfO}_{2}$ indicates that it should be capable of accurately predicting $\mathrm{HfO}_{2}$ surface properties. We emphasize that the purpose of this work is to determine surface properties of the perfect cut crystal. That is, we do not consider complex or extended reconstructions. The surface models we study include an integer number of $\mathrm{HfO}_{2}$ formula units and are thus stoichiometric. We follow the general rules provided by Christensen and Carter $^{19}$ and design slabs to model surfaces with maximum surface compactness and minimized coordination loss. We have also avoided generating surface models that are significantly polar and thus artificially stable due to long-range electrostatic forces.

Because monoclinic surfaces are more complex than other phases, the choice of a given surface index does not univocally define the surface structure in an obvious way. For our purpose, a slab corresponding to a given Miller face has an integer number of planes such that it is parallel to the surface and has a center of symmetry at the slab center. Consequently, it can be mapped onto all parallel planes below it by applying symmetry operators such as translations, screw axes or glide planes and thus the resulting slab has no dipole moment. The unrelaxed slabs have been cut from the bulk crystal, where bulk structures have been fully relaxed with respect to intracell and unit cell degrees of freedom. We have performed surface relaxations of all nine inequivalent lowindex faces of $m$ - $\mathrm{HfO}_{2}$. All atoms in the slab have been allowed to relax, although both sides of the slab remain equivalent as they are related by an inversion, or a mirror/ glide plane, located at the center of the slab. In these calculations we do not allow the slab unit cell to relax. In Figs. 1 and 2 we only report the atomic displacements of the (001) and (111) surfaces with respect to slab thickness as these faces were most prominent during the growth of $\mathrm{HfO}_{2}{ }^{14-17}$

In Figs. 1(a) and 1(b) we show the unrelaxed and relaxed (001) surfaces of a four layered slab. As can be seen in Fig. 1(a), there are two inequivalent surface hafnium atoms and 
TABLE I. Calculated structural parameters for three $\mathrm{HfO}_{2}$ phases. Lattice parameters $a, b, c$ are $\AA, \beta$ in degrees and $V$ (volume per formula) in $\AA^{3}$. Internal coordinates $x, y$, and $z$ are unitless.

\begin{tabular}{|c|c|c|c|c|}
\hline & Present GGA & Previous $\mathrm{LDA}^{\mathrm{a}}$ & Previous GGA ${ }^{\mathrm{a}}$ & Expt. $^{\mathrm{b}}$ \\
\hline \multicolumn{5}{|l|}{ Cubic } \\
\hline Energy/molecule & -30.45 & $\cdots$ & $\cdots$ & $\cdots$ \\
\hline$V$ & 32.11 & 31.95 & 36.15 & 32.77 \\
\hline$a$ & 5.045 & 5.248 & 5.04 & 5.08 \\
\hline \multicolumn{5}{|l|}{ Tetragonal } \\
\hline Energy/molecule & -30.51 & $\cdots$ & $\cdots$ & $\cdots$ \\
\hline$V$ & 32.69 & 32.77 & 37.74 & \\
\hline$a$ & 3.565 & 5.056 & 5.299 & \\
\hline$c$ & 5.146 & 5.127 & 5.373 & \\
\hline \multicolumn{5}{|l|}{ Monoclinic } \\
\hline Energy/molecule & -30.69 & $\cdots$ & $\cdots$ & $\cdots$ \\
\hline$V$ & 34.10 & 34.35 & 38.01 & 34.58 \\
\hline$a$ & 5.079 & 5.106 & 5.291 & 5.117 \\
\hline$b$ & 5.177 & 5.165 & 5.405 & 5.175 \\
\hline$c$ & 5.250 & 5.281 & 5.366 & 5.220 \\
\hline$\beta$ & 99.24 & 99.35 & 97.92 & 99.22 \\
\hline$x_{\mathrm{Hf}}$ & 0.277 & 0.280 & 0.276 & 0.276 \\
\hline$y_{\mathrm{Hf}}$ & 0.042 & 0.043 & 0.039 & 0.040 \\
\hline$z_{\mathrm{Hf}}$ & 0.207 & 0.209 & 0.209 & 0.208 \\
\hline$x_{\mathrm{O} 1}$ & 0.075 & 0.076 & 0.089 & 0.074 \\
\hline$y_{\mathrm{O} 1}$ & 0.343 & 0.346 & 0.367 & 0.332 \\
\hline$z_{\mathrm{O} 1}$ & 0.336 & 0.337 & 0.317 & 0.347 \\
\hline$x_{\mathrm{O} 2}$ & 0.446 & 0.447 & 0.447 & 0.449 \\
\hline$y_{\mathrm{O} 2}$ & 0.759 & 0.759 & 0.762 & 0.758 \\
\hline$z_{O 2}$ & 0.481 & 0.483 & 0.488 & 0.480 \\
\hline
\end{tabular}

Reference 29.

${ }^{b}$ Reference 10 for cubic and Ref. 32 for monoclinic.

two surface oxygen atoms. These inequivalent atoms are placed at different $z$ planes in the unrelaxed surface. However, upon relaxation these inequivalent atoms displace to almost align themselves in same $z$ plane. Figure 1(c) shows the quantitative displacements of inequivalent surface atoms for each direction. Both surface hafnium atoms move out of plane, however the second Hf atom [Hf2 in Fig. 1(a)] moves about $0.2 \AA$ more in the $z$ direction than the first Hf atom [Hf1 in Fig. 1(a)] in order to place itself in the same plane. The displacement of $\mathrm{O} 1$ along the $z$ direction is negligible; however, the second oxygen $(\mathrm{O} 2)$ is displaced by $\sim 0.5 \AA$ out of plane. Hence, the maximum displacement during relaxation is not necessarily exhibited by the outermost atoms. Furthermore, the displacement of atoms is not restricted to vertical movement, but atoms can also relax laterally. For example, $\mathrm{O} 1$ shifts almost by $0.4 \AA$ in the $x$ direction. Because of significant subsurface relaxation a slab with at least four layers is necessary to accurately describe the surfaces to predict their surface properties.

Similarly, in Figs. 2(a) and 2(b) we show the unrelaxed and relaxed $(\overline{1} 11)$ surfaces, respectively. The $(\overline{1} 11)$ face is a more complicated surface compared to the (001) surface. The (111) surface has four inequivalent surface Hf and sur- face $\mathrm{O}$ atoms. The surface features are not visibly different in Figs. 2(a) and 2(b). However, in Fig. 2(c) we quantify the displacements of the surface atoms. Like the (001) surface, most surface atoms show out of plane relaxations. There are also significant lateral displacements of $\mathrm{O}$ atoms in the relaxed (001) structure.

\section{Surface energy}

The surface energy, $E_{\text {surf }}(n)$, for a system comprising $n$ layers is defined as

$$
E_{\text {surf }}(n)=\frac{E_{\text {tot }}(n)-E_{\text {bulk }}(n)}{2 A},
$$

where $E_{\text {tot }}(n)$ and $A$ are the total energy and total surface area per molecular $\left(\mathrm{HfO}_{2}\right)$ unit, respectively. ${ }^{31} E_{\text {bulk }}$ refers to the energy of the bulk monoclinic system containing the same number of molecular units as the slab. Since the slab has two surfaces, the energy difference is normalized by twice the area of each surface in Eq. (1). In Figs. 1(d) and 2(d) we show surface energy as a function of the number of layers. In Table II we have also summarized our results for all of the nine inequivalent faces. A useful tool in the analysis of re- 


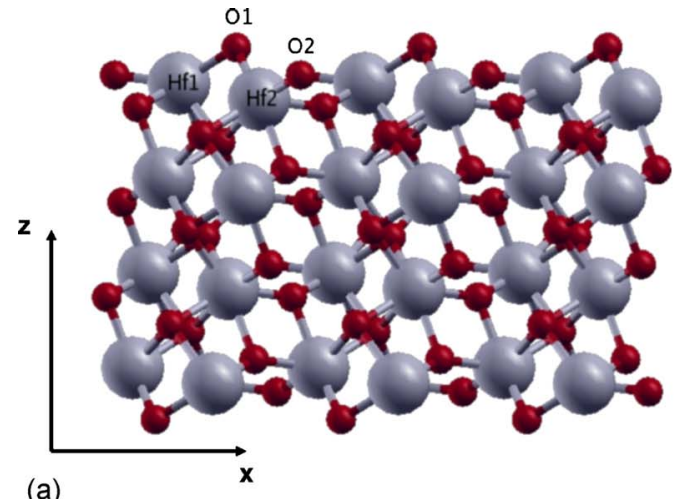

(a)

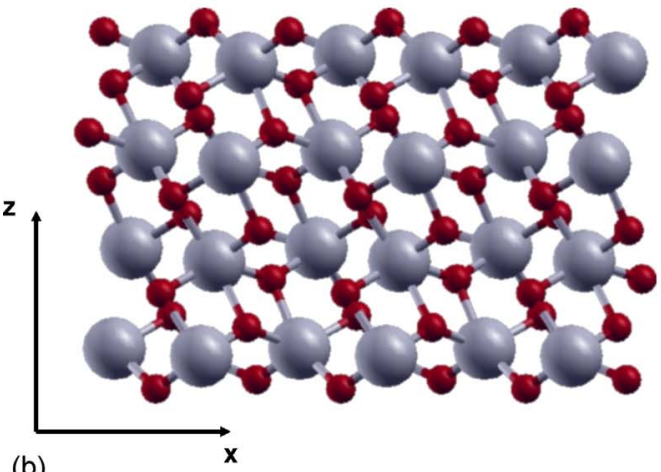

(b)

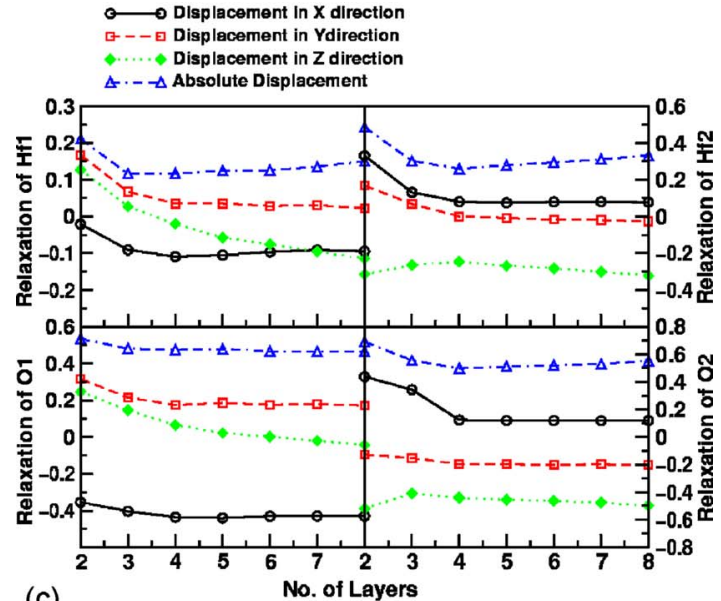

(c)

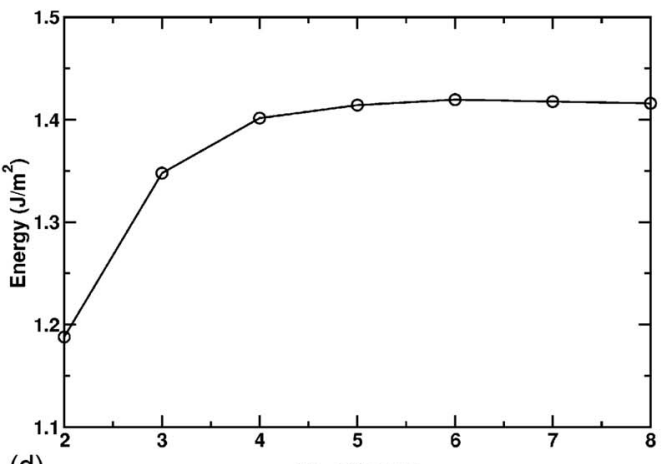

(d)

No. of Layers

FIG. 1. (Color online) (a) Unrelaxed and (b) relaxed (001) structure of the four-layer surface model of monoclinic $\mathrm{HfO}_{2}$. The surface atoms (Hf and $\mathrm{O}$ atoms) are indicated. (c) Calculated displacements of surface $\mathrm{Hf}$ and $\mathrm{O}$ atoms as a function of slab thickness. (d) Variation of surface energy as a function of slab thickness. laxation is the root mean squared (rms) ionic displacement reported in Table II. The rms ionic displacement is the average displacement of all $\mathrm{N}$ atoms in each slab relative to the geometrical center of mass $\Delta \mathbf{r}_{\text {c.m }}$ :

$$
\Delta \mathbf{r}_{r m s}=\frac{\sqrt{\sum_{i=1}^{N}\left(\Delta \mathbf{r}_{i}-\Delta \mathbf{r}_{c . m .}\right)^{2}}}{N} .
$$

This quantity will tend to decrease with increasing slab thickness because bulk ions have small or vanishing displacement upon relaxation. However, comparison of atomic displacement is a meaningful measure of relaxation as we have chosen all slabs to have approximately the same thickness of nearly $8 \AA$.

The (111) and (111) surfaces are the most stable faces of the monoclinic phase. These faces are also the ones corresponding to the most intense peaks in the XRD of various ALD films grown by various groups. ${ }^{14,15,18}$ However, some groups observed the peak corresponding to the (111) surface as the most intense peak along with that of the (001) face. ${ }^{4,16}$

This can be explained if the (111) and (111) surfaces are ascribed as the thermodynamically favored surfaces while the (001) face could correspond to the kinetically favored surface, We predict a surface stability order for the surfaces we consider very similar to that predicted for the $\mathrm{ZrO}_{2}$ surface.

with a few exceptions. ${ }^{19}$ The predicted order of stability for $\mathrm{ZrO}_{2}$ is $(\overline{1} 11)>(\overline{1} 01)>(111)>(110)>(011)>(001)$ $>(100)>(101)>(010)$, whereas in $\mathrm{HfO}_{2}$ we predict the stability order as $(\overline{1} 11)>(111)>(\overline{101})>(110)>(001)>(011)$ $>(101)>(100)>(010)$. However, the magnitude of relaxation energy for the $\mathrm{HfO}_{2}$ surface is larger than that of the $\mathrm{ZrO}_{2}$ surface. ${ }^{19}$

The surface energies reported in Table II indicate that the surface energy is highly anisotropic with a strong correlation between unrelaxed and relaxed surface energies. Consequently, the ordering of the unrelaxed surface stabilities is similar to that of the relaxed surface energies. The relaxation energy of the surfaces ranges from $23 \%$ to $36 \%$ of the total surface energy. We obtain a slight correlation between surface energies and the rms ionic relaxations.

\section{Electronic structure}

In this section we discuss the electronic properties of the surfaces. In Fig. 3, we report the bulk total density of states (DOS) obtained for the monoclinic crystal along with the partial DOS contributed by the three inequivalent atoms of the unit cell (one Hf atom and two $\mathrm{O}$ atoms). The contribution to the DOS from Hf comes from the $5 p$ and $5 d$ electrons, whereas for $\mathrm{O}$, the $2 s$ and $2 p$ electrons contribute. The total DOS is composed of two valence bands, a lower narrow band lying at about $-16.5 \mathrm{eV}$ composed of mainly $\mathrm{O} 2 \mathrm{~s}$ states and an upper band lying between -6.6 and $0.0 \mathrm{eV}$ mainly arising from $\mathrm{O} 2 p$ states, with a significant contribution of the Hf $5 d$ orbitals. Note that the two inequivalent atoms have noticeably different DOS features, as expected from their different environments. The upper valence band is 


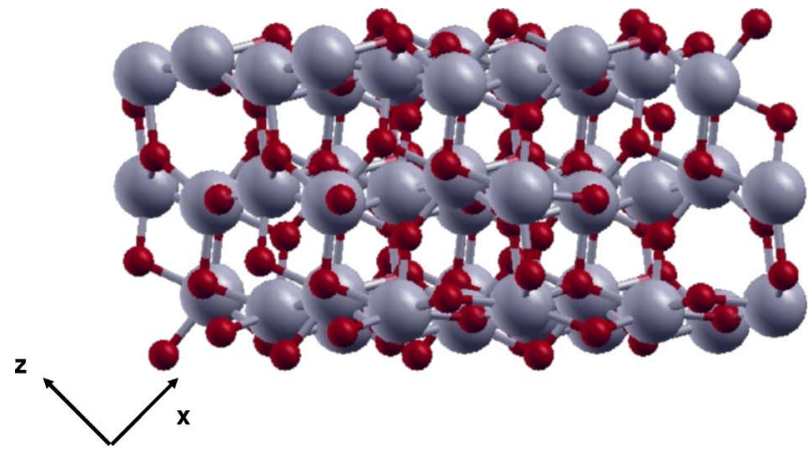

(a)

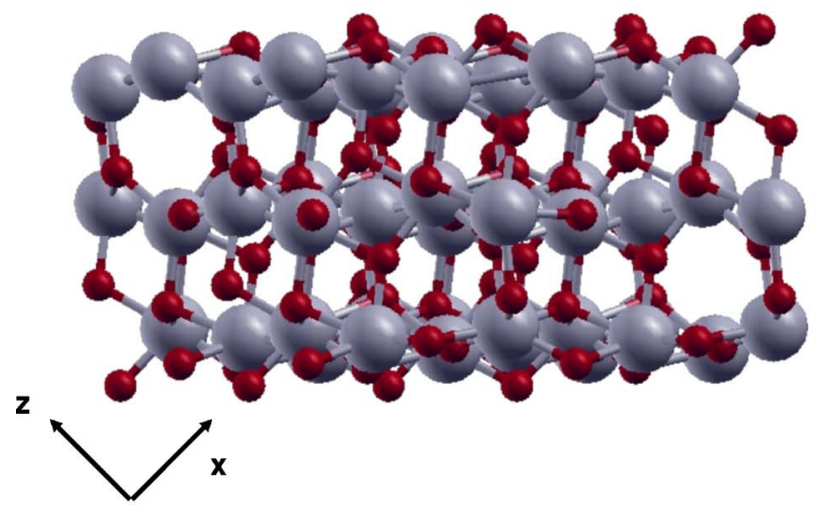

(b)

FIG. 2. (Color online) (a) Unrelaxed and (b) relaxed (111) structure of the three-layer surface model of monoclinic $\mathrm{HfO}_{2}$. (c) Calculated displacements of surface hafnium and oxygen atoms as a function of slab thickness. (d) Variation of the surface energy as a function of slab thickness.

separated by an energy gap of $3.8 \mathrm{eV}$ from a conduction band composed of Hf $5 d$ character. This is in agreement with previous theoretical studies ${ }^{33-35}$ as well as with the photoemission and inverse photoemission data obtained from $\mathrm{HfO}_{2}$ deposited on $\mathrm{SiO}_{x} \mathrm{~N}_{y} / p \mathrm{Si}$, which showed that the valence band mainly consists of $\mathrm{O} 2 p$ nonbonding orbitals of $\pi$ symmetry while the conduction band is primarily Hf $5 d$-like

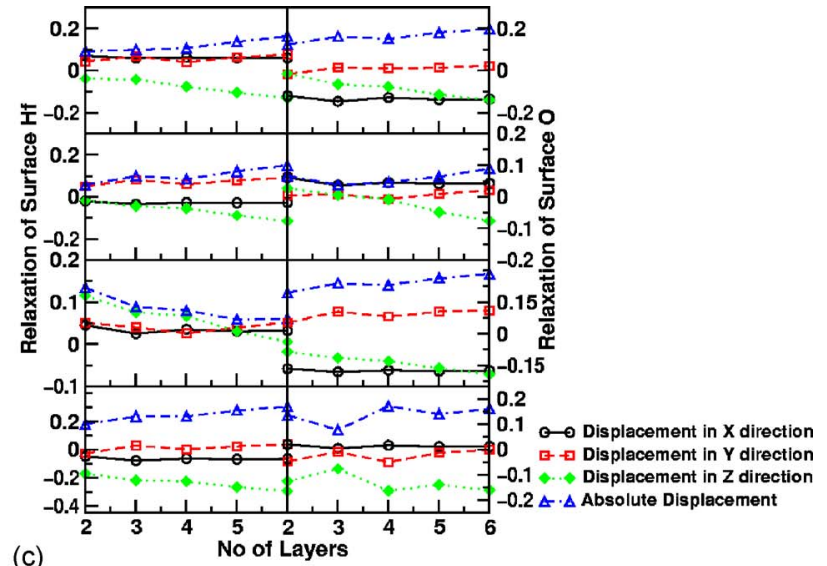

(c)

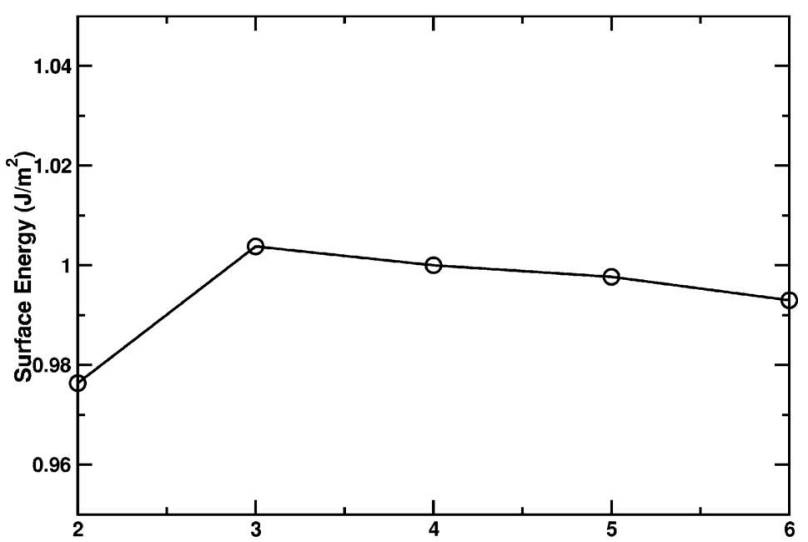

(d) nonbonding orbitals. ${ }^{35}$ However, our calculations show that the upper valence band exhibits mixing of the $\mathrm{O} 2 p$ and $\mathrm{Hf}$ $5 d$ states, indicating covalency of the Hf-O bonds.

In Fig. 4 we report the total DOS of both the unrelaxed and relaxed (111) surfaces as well as that of the monoclinic crystal. For the sake of comparison, the energy values of the

TABLE II. Surface energies for the nine inequivalent low-index faces of monoclinic $\mathrm{HfO}_{2}$.

\begin{tabular}{ccccc}
\hline \hline & \multicolumn{2}{c}{ Surface energy $\left(\mathrm{J} / \mathrm{m}^{2}\right)$} & & \%Relaxation \\
Energy & Unrelaxed & & $\begin{array}{c}\text { rms ionic } \\
\text { relaxation } \\
\AA\end{array}$ \\
\cline { 2 - 3 } Face & Relaxed & 1.460 & 32 & 0.026 \\
$(\overline{1} 11)$ & 0.993 & 1.562 & 23 & 0.023 \\
$(111)$ & 1.199 & 1.858 & 29 & 0.033 \\
$(101)$ & 1.322 & 2.043 & 32 & 0.030 \\
$(001)$ & 1.388 & 2.169 & 35 & 0.060 \\
$(011)$ & 1.416 & 2.100 & 29 & 0.024 \\
$(101)$ & 1.484 & 2.412 & 36 & 0.047 \\
$(100)$ & 1.550 & 2.165 & 23 & 0.021 \\
$(010)$ & 1.667 & 2.782 & 33 & 0.040 \\
\hline \hline
\end{tabular}




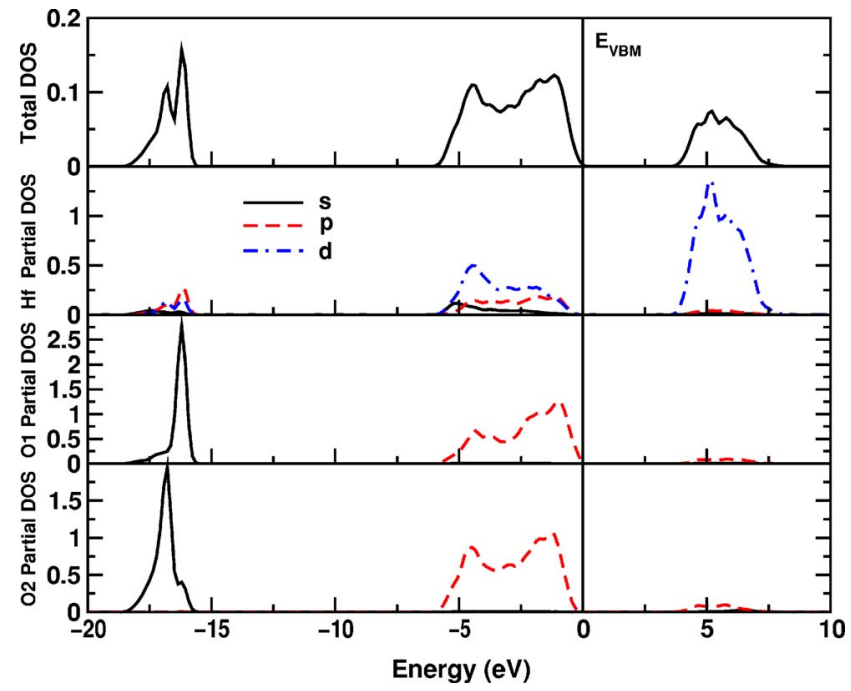

FIG. 3. (Color online) Total and partial DOS of bulk monoclinic $\mathrm{HfO}_{2} . E_{\mathrm{VBM}}$ represents the maximal energy of the valence band.

DOS of the surfaces in these plots have been shifted in order to align their valence band maxima with that of the bulk. Henceforth all comparisons will be made based on energies relative to the valence band maximum of the monoclinic bulk crystal. We do not present the calculated DOS of the other eight surfaces because we found them to exhibit similar features. The effect of relaxation of the slab on the total DOS is significant. Although the predicted band gaps may not be reliable (according to the well-known DFT underestimation), trends in the predicted gap should be meaningful. As can be observed, the upper valence band of the bulk and relaxed (111) surface look quite similar to each other while for the unrelaxed surface some states are pushed upwards into the lower part of the gap. As the zero of energy is referenced to the highest of these states it appears as though the entire DOS of the unrelaxed surface is shifted towards lower energy. The unrelaxed surface has a smaller band gap of $2.1 \mathrm{eV}$ compared to the bulk band gap of $3.8 \mathrm{eV}$. We predict two surface states near -15.0 and $0.1 \mathrm{eV}$ for the unrelaxed sur-

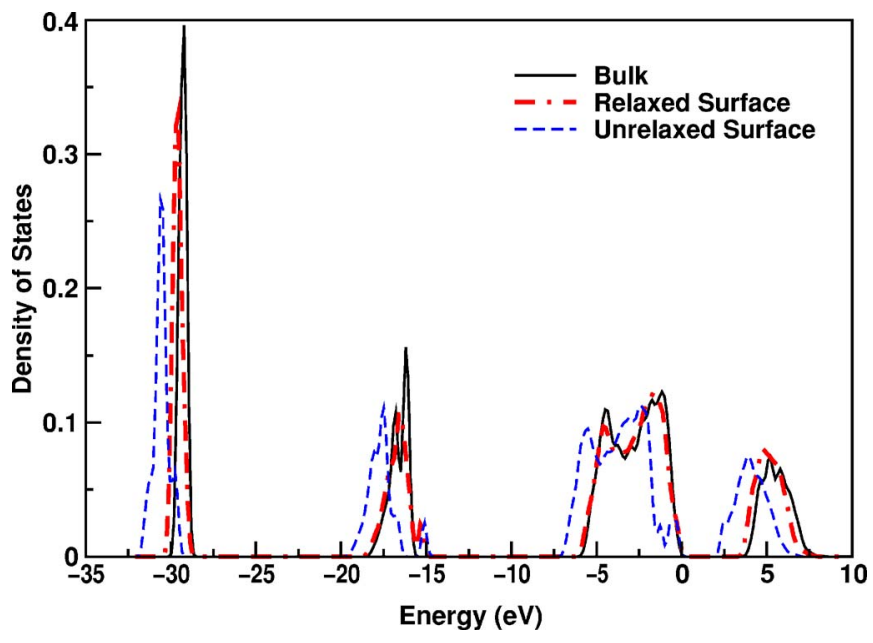

FIG. 4. (Color online) Total DOS of bulk, unrelaxed and relaxed (111) surfaces of monoclinic $\mathrm{HfO}_{2}$. face corresponding, respectively, to the $2 s$ and $2 p$ states of surface oxygen. Upon relaxation of the slab, the latter disappears, however the former state is retained. The DOS of the relaxed slab is almost identical to the DOS of the bulk except for one feature at $-15.0 \mathrm{eV}$. A more extensive analysis of the partial DOS shows that these extra surface states arise from an oxygen $2 s$ orbital corresponding to one of the surface $\mathrm{O}$ atoms. The band gap of the relaxed surface is approximately $0.5 \mathrm{eV}$ lower than that of the bulk. These features are very similar to those predicted for each of the other eight monoclinic surfaces.

\section{CONCLUSIONS}

In summary, we have predicted structural and electronic properties of different $m$-HfO 2 surfaces. Our GGA based DFT results are in excellent agreement with the existing experimental results of structural bulk properties. Calculated surface energies demonstrate that there is a strong correlation between unrelaxed and relaxed surface energies. We also found a weak correlation between surface energies and rms ionic relaxations. We predict that relaxations are not only limited to the outer surface atoms, but that subsurface atoms undergo significant relaxation. The calculated surface energies predict that the (111) and (111) surfaces are the most stable surfaces for the $m$ - $\mathrm{HfO}_{2}$ system in agreement with the appearance of these surfaces in ALD grown $\mathrm{HfO}_{2}$ films. However, the surface energy of the (001) face, which is observed in some of the ALD grown films, is estimated to be about $0.7 \mathrm{~J} / \mathrm{m}^{2}$ larger than the most stable surfaces suggesting that such a surface could be kinetically favored under given experimental conditions. Analysis of the partial DOS of the bulk phase indicates that there is a noticeable hybridization of $\mathrm{O} 2 p$ and $\mathrm{Hf} 5 d$ states, indicating a significant covalent contribution to the Hf-O bond. The electronic DOS of the relaxed and unrelaxed surfaces highlights the importance of surface relaxations and describes the nature of surface dominant states. For instance, we predict a prominent surface state near $-15.0 \mathrm{eV}$ contributed by surface $\mathrm{O} 2 s$ orbitals.

The present work is intended as a preliminary step towards the understanding of $\mathrm{HfO}_{2}$ surfaces and their reactivity which we hope will ultimately lead to a better understanding of the principles that govern the surface chemistry responsible for the ALD of $\mathrm{HfO}_{2}$ films. A subsequent publication will provide an in depth analysis of the surface reactions of ALD precursors on these relaxed monoclinic surfaces.

\section{ACKNOWLEDGMENTS}

We gratefully acknowledge the support of the Stanford Initiative for Nanoscale Materials and Processing and the Materials Structures and Devices SRC/DARPA MARCO Center. This work was partially supported by the Spanish Ministerio de Educación y Ciencia, and the European FEDER, Project No. MAT2005-01872. J.F.S. thanks the Ministerio de Educación y Ciencia, the Universidad de Sevilla and Stanford University for supporting his sabbatical stay at Stanford. 
*On leave from Departamento de Química Fisica, Facultad de Quimica, E-41012 Sevilla, Spain

${ }^{1}$ P. A. Packan, Science 285, 2079 (1999).

${ }^{2}$ C. T. Kuo, R. Kwor, and K. M. Jones, Thin Solid Films 213, 257 (1992)

${ }^{3}$ M. Ritala, M. Leskela, L. Niinisto, T. Prohaska, G. Friedbacher, and M. Grasserbauer, Thin Solid Films 250, 72 (1994).

${ }^{4}$ J. Aarik, A. Aidla, A. A. Kiisler, T. Uustare, and V. Sammelselg, Thin Solid Films 340, 110 (1999).

${ }^{5}$ H. O. Sankur and W. Gunning, Appl. Opt. 28, 2806 (1989).

${ }^{6}$ M. Reisse, B. Keiper, S. Weissmantel, H. Johansen, R. Scholz, and T. Martini, Thin Solid Films 241, 119 (1994).

${ }^{7}$ M. Alvisi, S. Scaglione, S. Martelli, A. Rizzo, and L. Vasanelli, Thin Solid Films 354, 19 (1999).

${ }^{8}$ M. Gilo and N. Croitoru, Thin Solid Films 350, 203 (1999).

${ }^{9}$ H. Ibegazene, S. Alperine, and C. Diot, J. Mater. Sci. 30, 938 (1995).

${ }^{10}$ J. Wang, H. P. Li, and R. Stivens, J. Mater. Sci. 27, 5397 (1992).

${ }^{11}$ M. H. Cho, Y. S. Roh, C. N. Whang, K. Jeong, S. W. Nahm, D. H. Ko, J. H. Lee, N. I. Lee, and K. Fujihara, Appl. Phys. Lett. 81, 472 (2002).

${ }^{12}$ R. R. Manory, T. Mori, I. Shimizu, S. Miyake, and G. Kimmel, J. Vac. Sci. Technol. A 20, 549 (2002).

${ }^{13}$ W. Zhu, T. P. Ma, T. Tamagawa, Y. Di, J. Kim, R. Carruthers, M. Gibson, and T. Furukawa, Tech. Dig. - Int. Electron Devices Meet. 464, (2001).

${ }^{14}$ M. Y. Ho, H. Gong, G. D. Wilk, B. W. Busch, M. L. Green, P. M. Voyles, D. A. Muller, M. Bude, W. H. Lin, A. See., M. E. Loomans, S. K. Lahiri, and P. T. Räisänen, J. Appl. Phys. 93, 1477 (2003).

${ }^{15}$ D. Triyoso, R. Liu, D. Roan, M. Ramon, N. V. Edwards, R. Gregory, D. Werho, J. Kulik, G. Tam, E. Irwin, X. D. Wang, L. B. La, C. Hobbs, R. Garcia, J. Baker, B. E. White, and P. Tobin, J.
Electrochem. Soc. 151, 220 (2004).

${ }^{16}$ J. Aarik, A. Aidla, H. Mändar, V. Sammelselg, and T. Uustare, J. Cryst. Growth 220, 105 (2000).

${ }^{17}$ A. Aarik, H. Mändar, M. Kirm, and L. Pung, Thin Solid Films 466, 41 (2004).

${ }^{18}$ K. Kukli, J. Aarik, M. Ritala, T. Uustare, T. Sajavaara, J. Lu, J. Sundqvist, A. Aidla, L. Pung, A. Harsta, and M. Lesela, J. Appl. Phys. 96, 5298 (2004).

${ }^{19}$ A. Christensen and E. A. Carter, Phys. Rev. B 58, 8050 (1998).

${ }^{20}$ G. Kresse and J. Hafner, Phys. Rev. B 47, 558 (1993).

${ }^{21}$ G. Kresse and J. Furthmuller, Comput. Mater. Sci. 6, 15 (1996).

${ }^{22}$ J. P. Perdew, J. Chevary, S. Vosko, K. Jackson, M. Pederson, D. Singh, and C. Fiolhais, Phys. Rev. B 46, 6671 (1992).

${ }^{23}$ J. P. Perdew, in Electronic Structure in Solids, edited by P. Ziesche and H. Eschrig (Akademie, Berlin 1991).

${ }^{24}$ P. E. Blöchl, Phys. Rev. B 50, 17953 (1994).

${ }^{25}$ J. Harris, Phys. Rev. B 31, 1770 (1985).

${ }^{26}$ S. Desgreniers and K. Lagarec, Phys. Rev. B 59, 8467 (1999).

${ }^{27}$ J. E. Lowther, J. K. Dewhurst, J. M. Leger, and J. Haines, Phys. Rev. B 60, 14485 (1999).

${ }^{28}$ R. Ruth and V. A. Patel, J. Am. Ceram. Soc. 56, 606 (1973).

${ }^{29}$ X. Zhao and D. Vanderbilt, Phys. Rev. B 65, 233106 (2002).

${ }^{30}$ Handbook of Chemistry and Physics, 85th ed. (CRC, Cleveland, 2004).

${ }^{31}$ S. P. Bates, G. Kresse, and M. J. Gillan, Surf. Sci. 385, 368 (1997).

${ }^{32}$ J. Adam and M. D. Rodgers, Acta Crystallogr. 12, 951 (1959).

${ }^{33}$ A. A. Demkov, Phys. Status Solidi B 226, 57 (2001).

${ }^{34}$ P. K. Boer and R. A. de Groot, J. Phys.: Condens. Matter 10, 10241 (1998).

${ }^{35}$ S. Sayan, T. Emge, E. Garfunkel, X. Zhao, L. Wielunski, R. A. Bartynski, D. Vanderbilt, J. S. Suehle, S. Suzer, and M. Banaszak-Holl, J. Appl. Phys. 96, 7485 (2004). 